Article

\title{
Eudragit S100 Coated Citrus Pectin Nanoparticles for Colon Targeting of 5-Fluorouracil
}

\author{
M. Biswaranjan Subudhi, Ankit Jain, Ashish Jain, Pooja Hurkat, Satish Shilpi, Arvind Gulbake \\ and Sanjay K. Jain * \\ Department of Pharmaceutical Sciences, Dr. Hari Singh Gour Central University, \\ Sagar 470 003, (MP), India; E-Mails: bisu_ai75@yahoo.co.in (M.B.S.); \\ ankitjainsagar@gmail.com (A.J.); ashish.g.jain@gmail.com (A.J.); pooja159@gmail.com (P.H.); \\ shilpisatish@gmail.com (S.S.); arvind.gulbake@gmail.com (A.G.) \\ * Author to whom correspondence should be addressed; E-Mail: drskjainin@gmail.com; \\ Tel.: +91-7582-225782; Fax: +91-7582-264236.
}

Academic Editor: Loo Say Chye Joachim

Received: 13 January 2015 / Accepted: 13 February 2015 / Published: 27 February 2015

\begin{abstract}
In the present study, Eudragit S100 coated Citrus Pectin Nanoparticles (E-CPNs) were prepared for the colon targeting of 5-Fluorouracil (5-FU). Citrus pectin also acts as a ligand for galectin-3 receptors that are over expressed on colorectal cancer cells. Nanoparticles (CPNs and E-CPNs) were characterized for various physical parameters such as particle size, size distribution, and shape etc. In vitro drug release studies revealed selective drug release in the colonic region in the case of E-CPNs of more than $70 \%$ after $24 \mathrm{~h}$. In vitro cytoxicity assay (Sulphorhodamine B assay) was performed against HT-29 cancer cells and exhibited 1.5 fold greater cytotoxicity potential of nanoparticles compared to 5-FU solution. In vivo data clearly depicted that Eudragit S100 successfully guarded nanoparticles to reach the colonic region wherein nanoparticles were taken up and showed drug release for an extended period of time. Therefore, a multifaceted strategy is introduced here in terms of receptor mediated uptake and $\mathrm{pH}$-dependent release using E-CPNs for effective chemotherapy of colorectal cancer with uncompromised safety and efficacy.
\end{abstract}

Keywords: Eudragit S100; citrus pectin; 5-Fluorouracil; colon targeting; cancer 


\section{Introduction}

Colorectal cancer is the second leading cause of cancer-related mortality with about 655,000 deaths worldwide every year. Conventional therapies for colorectal cancer include surgery, chemotherapy, and radiation therapy. Conventional chemotherapy is not very effective as the drug does not reach the target site in effective concentrations. Thus, effective treatment demands an increased dose size, which may lead to undue side effects [1]. To overcome these situations, a colon specific drug delivery approach is employed to bring about favorable consequences i.e., minimized drug distribution to non-target cells in order to shun untoward effects, improved targeting ability to cancer cells, and controlled release (temporal effects) of cytotoxic drug to cancer cells in effective concentration. As a drug of choice, 5-fluorouracil (5-FU) is selected because of designated first line therapy for colorectal cancer [2].

In the past, a number of polymeric materials were used for the preparation of nanoparticles. Recently, polysaccharides have been investigated for the preparation of nanoparticles because of their excellent physicochemical properties and biocompatible nature which are beneficial for biomedical use [3-5]. Pectins are a family of complex polysaccharides that contain large amounts of poly-(d-galactouronic acid) bonded via $\alpha$-1,4-glycosidic linkage. They are heterogeneous moieties with respect to chemical structure and molecular weight [6] and can be classified into low methoxy (LM), high methoxy (HM) and amidated pectins. Pectins have a number of pharmaceutical applications and are presently considered as promising biodegradable carriers for colon-specific drug delivery for systemic action or topical treatment of diseases such as ulcerative colitis, Crohn's disease, and colon carcinomas, as indicated by a plethora of studies published over the last decennia [7-10]. Various techniques have been reported to manufacture the pectin-based drug delivery systems, especially ionotropic gelation and gel coating [11,12]. The most striking property of pectins for industrial applications is their gelling activity. Factors governing gelation and thereby influencing gel characteristics are the type and concentration of pectin, the modifications of hydroxyl group, the $\mathrm{pH}$ of pectin solution, the temperature and the presence of cations [13]. HM-pectin forms gels with sugar and acid. HM-pectin, unlike LM-pectin, does not contain sufficient acid groups to gel or precipitate with calcium ions. The mechanism of LM-pectin gelation relies mainly on the well-known "egg-box" model [14]. Amidation further improves the gelling potential of LM-pectin. Amidated pectins need less calcium to gel and are less prone to precipitation at high calcium levels [15]. When solution $\mathrm{pH}$ is raised, the polycarboxylate groups are ionized, and able to react with calcium ions to form calcium-pectinate gels. The interaction of calcium ions and the carboxylate groups of pectin results in intermolecular chelation leading to the formation of macromolecular aggregates [16]. This property of pectin was employed to form the nanoparticulate system. Various drug delivery approaches have been developed for colon-specific drug delivery, which include a $\mathrm{pH}$-sensitive system, a time-dependent system, pro-drugs, and a microflora-activated system to deliver therapeutic agents to the desired colonic sites; hence systemic drug absorption should be reduced as this leads to unwanted systemic side effects $[17,18]$.

Of all the systems formulated for colon-specific drug delivery, the pH-sensitive system and time-dependent system are mostly used [8-10]. The $\mathrm{pH}$ variation along the GI tract (GIT) is based on the strategy of using $\mathrm{pH}$ as a trigger to achieve drug release in the colon. The high individual 
variability together with similarity in $\mathrm{pH}$ between the small intestine and the colon make the site specificity of the $\mathrm{pH}$-dependent system not very reliable [11,12].

Eudragit S 100 is an anionic copolymer based on methacrylic acid and methyl methacrylate. The ratio of the free carboxyl groups to ester groups is approx. 1:2. It was selected for coating of citrus pectin nanoparticles due to its desirable attributes such as $\mathrm{pH}$ responsive nature i.e., no release of entrapped drug at low $\mathrm{pH}$ of the stomach as well as in the small intestine where the $\mathrm{pH}$ is almost neutral. In this way, it imparts a colon specific nature to the delivery system.

Thus, in the present project, citrus pectin and Eudragit S100 (pH-responsive enteric polymer) were chosen to fabricate nanoparticulate drug delivery systems for site-specific delivery of 5-FU for the effective treatment of colorectal cancer. The proposed delivery system is premised to protect the drug loss in the upper GI tract because of the deterrent nature of Eudragit S100 (ES) to the milieu of the upper GI tract and to deliver 5-FU upon reaching the colon due to the amiable $\mathrm{pH}$ of the colonic fluid. Moreover, citrus pectin being biodegradable in nature also acts as a ligand for the galectin-3 receptors which are over expressed on the colorectal cancer cells [19]. Thus, a multifaceted approach is conceived which includes biodegradability and colon selectivity in terms of receptor mediated uptake and $\mathrm{pH}$-dependent release of 5-FU using Eudragit S100 coated citrus pectin nanoparticles (E-CPNs) for a better treatment of colorectal cancer.

\section{Materials and Methods}

The 5-FU was received as a gift sample from Biochem (Mumbai, India). Eudragit S100 was procured as gift sample from Evonik Degussa India Pvt Ltd (Mumbai, India). Pectin (P9135, pectin from citrus peel, galacturonic acid $\mathrm{g} \geq 74.0 \%$ ) was purchased from Sigma-Aldrich (Mumbai, India). Pancreatin (from pig pancreas), pepsin (bovine) and dialysis membrane (Molecular weight cut-off $3.5 \mathrm{kD}$ ) were procured from Himedia (Mumbai, India). Pectinase was purchased from Loba Chemie (Mumbai, India). All other chemicals used were of analytical grade. Double distilled water (DDW) was used throughout the study wherever needed.

\subsection{Preparation of Citrus Pectin Nanoparticles (CPNs)}

Citrus pectin nanoparticles (CPNs) were prepared using the method reported by Yu et al. (2009) with suitable modifications [20]. Briefly, citrus pectin (100 mg) was added to $5 \mathrm{~mL}$ of DDW under constant mechanical stirring (Remi, Mumbai, India) for $1 \mathrm{~h}$ at $1000 \mathrm{rpm}$ and then immersed in a water bath at $55{ }^{\circ} \mathrm{C}$ for $30 \mathrm{~min}$ to obtain a solution with a viscosity of $80 \mathrm{cps}$, as measured by a rotational viscometer (Brookfield Digital DV-E Viscometer, Middleboro, MA, USA). Then, powdered 5-FU $\left(75 \mathrm{mg}\right.$ ) was added to the system maintained at $55^{\circ} \mathrm{C}$ with stirring $(1000 \mathrm{rpm}$ for $1 \mathrm{~h}$ ). After that, $1 \mathrm{~mL}$ of calcium hydroxide solution $(0.025 \mathrm{M})$ was added to the system drop-wise with continuous stirring for an additional $1 \mathrm{~h}$ to obtain a mixture with a viscosity of $90 \mathrm{cps}$. The system was kept stirring at $55{ }^{\circ} \mathrm{C}$ for $2 \mathrm{~h}$ and then cooled rapidly in a water bath to $20^{\circ} \mathrm{C}$. After $30 \mathrm{~min}$, the mixture was put into a dialysis bag and dialyzed against $500 \mathrm{~mL}$ of DDW at $20{ }^{\circ} \mathrm{C}$ for $24 \mathrm{~h}$ to obtain the drug-loaded nanoparticles (CPNs). These CPNs were then lyophilized (Freeze-dryer, Labconco, Kansas City, MI, USA) to obtain a dry form. 


\subsection{Coating of CPNs}

The coating of CPNs was performed by a simple solvent evaporation method as reported by Maestrelli et al. (2008) [21]. The enteric coating solution was composed of Eudragit S100 in acetone $(12 \% \mathrm{w} / \mathrm{v})$. Coating was obtained by dispersing $100 \mathrm{mg}$ of CPNs in coating solution with a core: coat ratio of 1:10 followed by solvent evaporation in a rotary evaporator (Super Fit, Ambala, India). The process was repeated until the desired amount of coating was achieved. Samples of coated nanoparticles (E-CPNs) were then dried and weighed (Table S1).

\subsection{Characterization of CPNs and E-CPNs}

\subsubsection{Particle Size}

The average particle size and polydispersity index of the nanoparticles (CPNs and E-CPNs) were determined by photon correlation spectroscopy using a Zetasizer DTS ver. 5.03 (Malvern Instrument, Worcestershire, England). The samples of nanoparticles dispersions were diluted to four times their volume with $0.05 \mathrm{M} \mathrm{NaCl}$. The particle size and PDI are represented by the average (diameter) of the Gaussian distribution function in the logarithmic axis mode.

\subsubsection{Zeta Potential}

The zeta potential of nanoparticles (CPNs and E-CPNs) was determined by measurement of the electrophoretic mobility applying the Helmholtz-Smoluchowsky equation. For measurement of zeta potential, Zetasizer DTS ver. 5.03 (Malvern Instrument, Worcestershire, UK) was used. Samples were adjusted to a conductivity of $80 \mu \mathrm{S} / \mathrm{cm}$ with a solution of $0.05 \mathrm{M} \mathrm{NaCl}$ at field strength of $20 \mathrm{~V} / \mathrm{cm}$.

\subsubsection{Particle Shape and Surface Morphology}

\section{Transmission Electron Microscopy}

The nanoparticles (CPNs and E-CPNs) were characterized for their shape by transmission electron microscopy (Philips Morgagni 268D, Eindhoven, Netherlands). The samples (10 $\mu \mathrm{L})$ were adhered to coated grids and negatively stained with $2 \%(\mathrm{w} / \mathrm{v})$ phosphotungstic acid for $90 \mathrm{~s}$, and then blot dried using filter paper. The grid was allowed to dry carefully in air, and samples were examined under transmission electron microscope at suitable magnification (Figure 1).

\section{Scanning Electron Microscopy}

The nanoparticles (CPNs and E-CPNs) were characterized for their morphology by scanning electron microscopy (LEO 435 VP, Eindhoven, The Netherlands). To prepare the samples, a double-adhesive tape was stuck to an aluminum stub and removed to obtain an adhesive coated aluminum stub. Then, 1-2 drops of nanoparticles dispersion were applied on the stub and dried overnight in a desiccator. The stubs were coated with gold to a thickness of $\sim 300 \AA$ under an argon atmosphere using a gold sputter coater in a high-vacuum evaporator. The coater was operated at 
0.1 torr (argon) for $90 \mathrm{~s}$ at an accelerating voltage of $15 \mathrm{kV}$. The coated samples were then randomly investigated using a scanning electron microscope at suitable magnification (Figure 1).

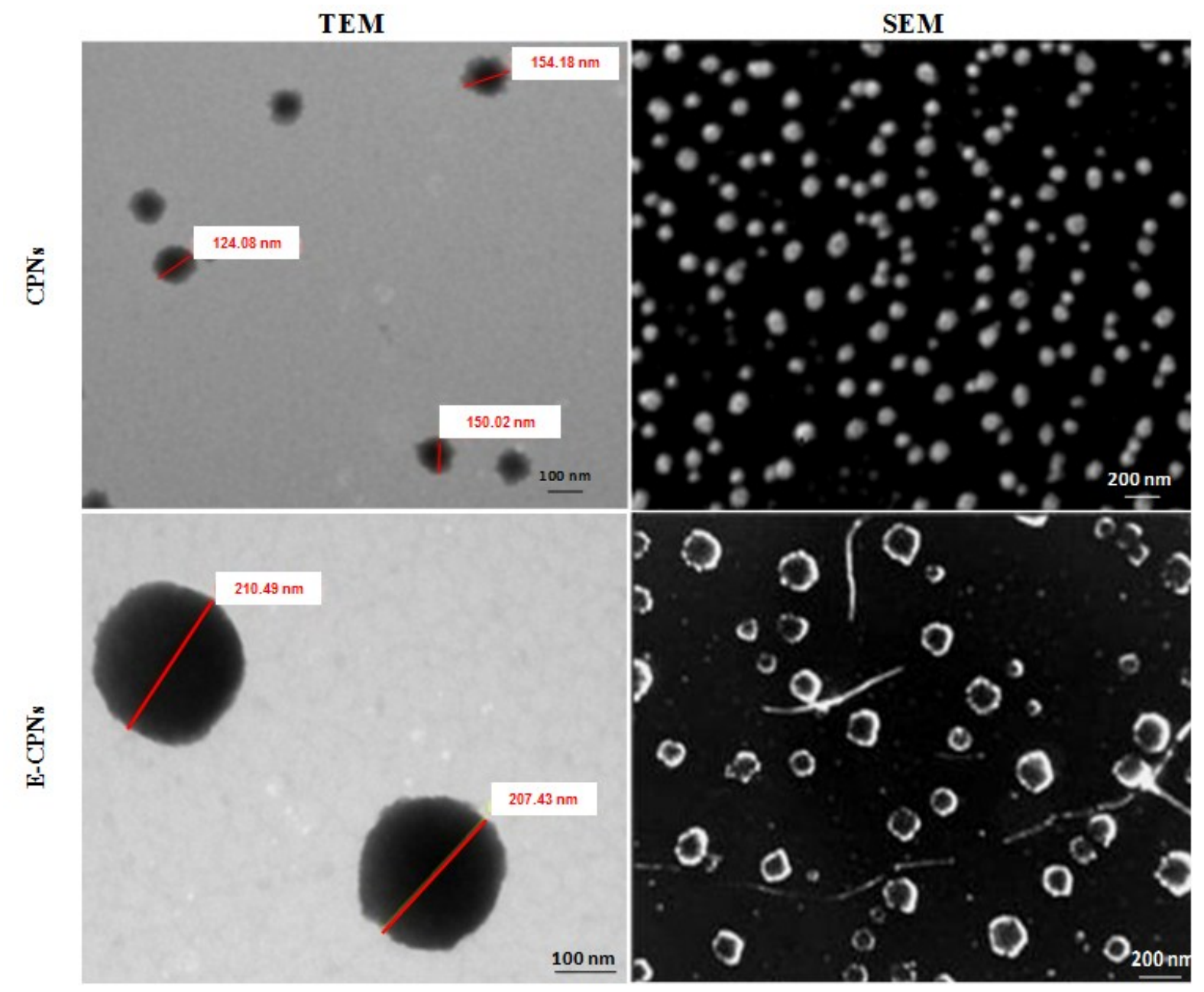

Figure 1. Transmission electron microscopy (TEM) and scanning electron microscopy (SEM) images of nanoparticles, i.e., Citrus pectin nanoparticles (CPNs) and Eudragit S100 coated citrus pectin nanoparticles (E-CPNs).

\subsubsection{Entrapment Efficiency}

The nanoparticles (CPNs and E-CPNs) weighing $50 \mathrm{mg}$ were digested in $5 \mathrm{~mL}$ of pectinase solution (120 FDU/mL, $\mathrm{pH} \mathrm{7.0)} \mathrm{for} 12 \mathrm{~h}$ [22]. The digested homogenate was centrifuged (Remi, Mumbai, India) at $3000 \mathrm{rpm}$ for $5 \mathrm{~min}$, and the supernatant was analyzed for 5-FU content using a double beam UV spectrophotometer at $\lambda_{\max } 266.0 \mathrm{~nm}$. The percent entrapment efficiency (\%EE) and percent drug loading $(\% \mathrm{DL})$ were calculated using the formulae (Table 1$)$ :

$$
\begin{gathered}
\% \mathrm{EE}=\frac{\text { Mass of drug in nanoparticles }}{\text { Mass of drug used in formulation }} \times 100 \\
\% \text { drug loading }(\mathrm{DL})=\frac{\text { Mass of drug in nanoparticles }}{\text { Mass of nanoparticles recovered }} \times 100
\end{gathered}
$$


Table 1. Characterization of nanoparticles.

\begin{tabular}{cccccc}
\hline $\begin{array}{c}\text { Formulation } \\
\text { code }\end{array}$ & $\begin{array}{c}\text { Particle size } \\
(\mathbf{n m})\end{array}$ & PDI & $\begin{array}{c}\text { Zeta potential } \\
(\mathbf{m V})\end{array}$ & $\begin{array}{c}\text { \% Entrapment } \\
\text { efficiency }\end{array}$ & $\begin{array}{c}\text { \% Drug } \\
\text { loading }\end{array}$ \\
\hline CPNs & $174.65 \pm 5.32$ & 0.095 & $-18.4 \pm 0.4$ & $38.75 \pm 0.74$ & $21.45 \pm 0.87$ \\
E-CPNs & $218.12 \pm 10.25$ & 0.117 & $-27.5 \pm 0.8$ & $35.15 \pm 0.52$ & $20.84 \pm 0.75$ \\
\hline
\end{tabular}

Values represent Mean $\pm \mathrm{SD}, n=3$.

\subsection{In vitro Release Study}

\subsubsection{In Simulated Gastric Fluids of Different $\mathrm{pH}$ Conditions}

In vitro drug release studies were carried out according to the Souder and Ellenbogen extraction technique with slight modifications [23]. The intention of using the simulated fluids of different $\mathrm{pH}$ was to mimic mouth-to-colon transit in the following manner: (a) 1-2 h: simulated gastric fluids (SGF) of $\mathrm{pH} 1.2$; (b) 3-4 h: mixture of simulated gastric and intestinal fluids of $\mathrm{pH} 4.5$; (c) 5-6 h: simulated intestinal fluids (SIF) of $\mathrm{pH} 6.8$; (d) 7-24 h: simulated colonic fluid (SCF) pH 7.0.

In vitro drug release patterns from nanoparticles (CPNs and E-CPNs) were studied using a dialysis bag [24]. Nanoparticles $(50 \mathrm{mg})$ taken in a dialysis bag (MWCO $3.5 \mathrm{kD}$ ) were placed in a beaker containing $100 \mathrm{~mL}$ of simulated fluids at $37 \pm 1{ }^{\circ} \mathrm{C}$ with slow magnetic stirring under perfect sink conditions and the fluids were changed as per the Souder and Ellenbogen scheme at a definite time point. Samples $(1 \mathrm{~mL})$ were withdrawn periodically and replaced with the same volume of fresh dissolution medium. The amount of drug released was determined using UV-spectroscopy at $\lambda_{\max }$ $266.0 \mathrm{~nm}$ and reported as the percent cumulative drug release in Figure 2.

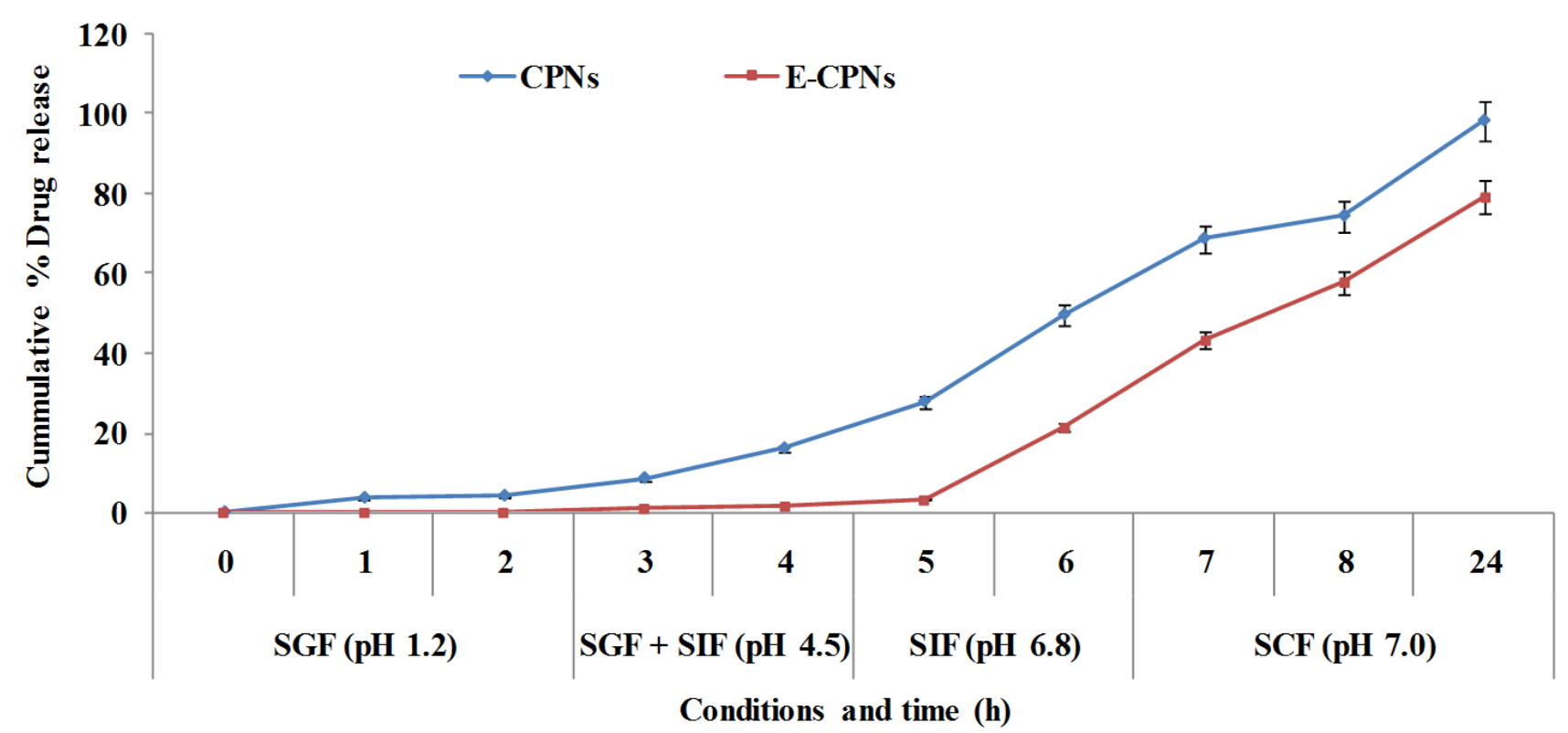

Figure 2. In vitro drug release profiles of CPNs \& E-CPNs in different simulated GI tract (GIT) fluids over a period of time (Mean $\pm \mathrm{SD}, n=3$ ). [GIT: Gastro-intestinal tract; SGF: Simulated gastric fluid; SIF: Simulated intestinal fluid; SCF: Simulated colonic fluid]. 


\subsubsection{In vitro Drug Release Study in the Presence and Absence of Rat Caecal Content}

To overcome the limitations of conventional dissolution tests for evaluating the performance of colon specific drug delivery systems triggered by colon specific bacterial flora, rat caecal content has been utilized as an alternative dissolution medium so called rat caecal content medium or simulated colonic fluid. This medium was prepared using the method as reported by Van den Mooter et al. (1994) [25]. Rats weighing 120-150 g were taken and maintained on normal diet. Citrus pectin solution $(1 \mathrm{~mL}, 2 \% \mathrm{w} / \mathrm{v})$ was administered through the oral route and this treatment was continued for seven days in order to induce the enzymes specific for the biodegradation of the pectin during its passage through the colon. Rats were sacrificed by spinal traction for $45 \mathrm{~min}$ prior to commencement of drug release studies. The abdomen was opened, the caecum was traced, ligated at both ends, dissected, and immediately transferred into PBS ( $\mathrm{pH}$ 7.0) bubbled previously with $\mathrm{CO}_{2}$. The caecal bags were opened and their contents were individually weighed, pooled, and suspended in the buffer continuously bubbled with $\mathrm{CO}_{2}$. They were finally added to the dissolution media to give a final caecal dilution of $2 \% \mathrm{w} / \mathrm{v}$. The suspension was then filtered through cotton wool and sonicated using a probe sonicator (Lark innovative technology, Chennai, India) at $950 \mathrm{~W}$ for $5 \mathrm{~min}$ at $4{ }^{\circ} \mathrm{C}$ to disrupt the bacterial cells. After sonication, the mixture was centrifuged at $2000 \mathrm{rpm}$ for $20 \mathrm{~min}$. As the environment in caecum is naturally anaerobic, all the operations were carried out under $\mathrm{CO}_{2}$ atmosphere [26].

Drug release studies of nanoparticles (CPNs and E-CPNs) in the presence and absence of rat caecal content were carried out in sealed glass vials with $10 \mathrm{~mL}$ of dissolution medium maintained at $37 \pm 2{ }^{\circ} \mathrm{C}$ under slow magnetic stirring. $50 \mathrm{mg}$ of nanoparticles was placed in the dissolution medium (PBS, pH 7.0) containing $2 \%$ rat caecal contents. Samples $(0.2 \mathrm{~mL})$ were withdrawn after a fixed time interval of $1,2,3 \ldots 8$ and $24 \mathrm{~h}$, volumes were made up to $10 \mathrm{~mL}$ and the same volume of dissolution medium was replaced with PBS ( $\mathrm{pH} 7.0)$. The withdrawn samples were centrifuged at $2000 \mathrm{rpm}$ for $10 \mathrm{~min}$ and the supernatant was filtered through Whatmann filter paper followed by estimation of 5-FU spectrophotometrically (Figure 3).

\subsection{In vitro Cytotoxicity Studies}

In vitro cytotoxicity potentials of free 5-FU solution and nanoparticles (CPNs and E-CPNs) suspension against HT-29 cells were compared using sulphorhodamine B assay (SRB assay) [27,28]. The cell lines were grown in RPMI 1640 medium containing $10 \%$ fetal bovine serum and $2 \mathrm{mM}$ L-glutamine. For the present screening experiment, cells were inoculated into 96 well microtiter plates in $100 \mu \mathrm{L}$ at plating densities, depending on the doubling time of individual cell lines. After cell inoculation, the microtiter plates were incubated at $37{ }^{\circ} \mathrm{C}, 5 \% \mathrm{CO}_{2}, 95 \%$ air, and $100 \%$ relative humidity for $24 \mathrm{~h}$ prior to addition of the formulation.

After $24 \mathrm{~h}$, one 96 well plate containing $5 \times 10^{3}$ cells/well was fixed in situ with trichloroacetic acid to represent a measurement of the cell population at the time of addition of the drug (Tz). Experimental samples (free drug and nanoparticles) were initially solubilized in dimethylsulfoxide at $100 \mathrm{mg} / \mathrm{mL}$ and diluted to $1 \mathrm{mg} / \mathrm{mL}$ using water and frozen prior to use. At the time of drug addition, an aliquot of frozen concentrate $(1 \mathrm{mg} / \mathrm{mL})$ was thawed and diluted to $100 \mu \mathrm{g} / \mathrm{mL}, 200 \mu \mathrm{g} / \mathrm{mL}, 400 \mu \mathrm{g} / \mathrm{mL}$ and 
$800 \mu \mathrm{g} / \mathrm{mL}$ with complete medium containing test article. Aliquots of $10 \mu \mathrm{L}$ of these different drug dilutions were added to the appropriate microtiter wells already containing $90 \mu \mathrm{L}$ of medium, resulting in the required final drug concentrations i.e. $10 \mu \mathrm{g} / \mathrm{mL}, 20 \mu \mathrm{g} / \mathrm{mL}, 40 \mu \mathrm{g} / \mathrm{mL}$ and $80 \mu \mathrm{g} / \mathrm{mL}$.

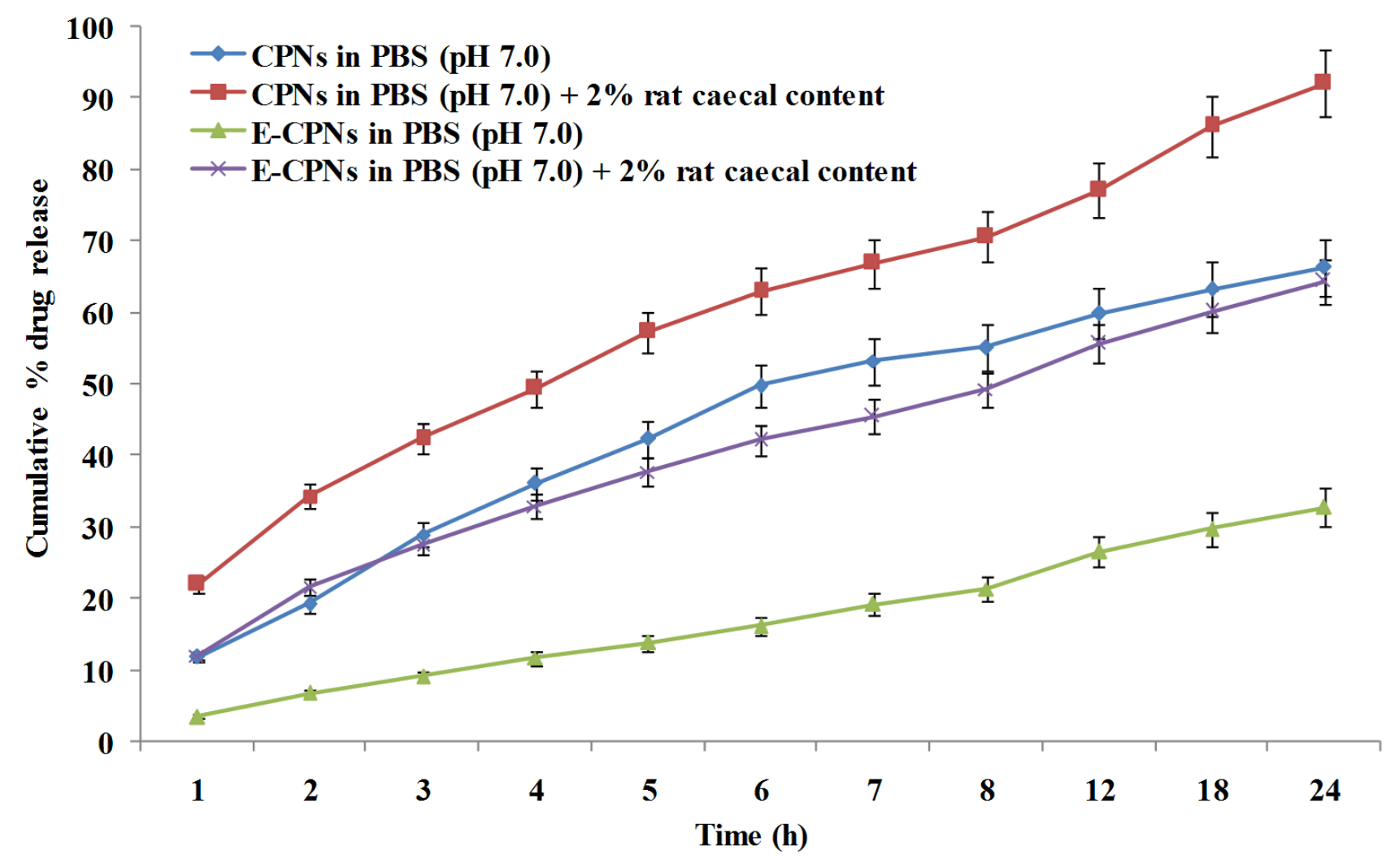

Figure 3. Cumulative \% drug release profiles of CPNs and E-CPNs at various conditions $($ Mean $\pm \mathrm{SD}, n=3)$.

\section{Endpoint Measurement}

After addition of different formulations, plates were incubated at standard conditions for $48 \mathrm{~h}$ and assay was terminated by the addition of cold TCA. Cells were fixed in situ by the gentle addition of $50 \mu \mathrm{L}$ of cold $30 \%(\mathrm{w} / \mathrm{v})$ TCA (final concentration, 10\% TCA) and incubated for 60 min at $4{ }^{\circ} \mathrm{C}$. The supernatant was discarded; the plates were washed five times with DDW and air dried. Sulforhodamine B $(\mathrm{SRB})$ solution $(50 \mu \mathrm{L})$ at $0.4 \%(\mathrm{w} / \mathrm{v})$ in $1 \%$ acetic acid was added to each of the wells, and plates were incubated for $20 \mathrm{~min}$ at room temperature. After staining, unbound dye was recovered and the residual dye was removed by washing five times with $1 \%$ acetic acid. The plates were air dried. Bound stain was subsequently eluted with $10 \mathrm{mM}$ trizma base, and the absorbance was read on a plate reader at a wavelength of $540 \mathrm{~nm}$ with $690 \mathrm{~nm}$ reference wavelength [29].

Percent growth was calculated on a plate-by-plate basis for test wells relative to control wells. Percent growth was expressed as the ratio of the average absorbance of the test well to the average absorbance of the control wells $\times 100$. Using the six absorbance measurements [time zero $(\mathrm{Tz})$, control growth (C), and test growth in the presence of drug at the four concentration levels (Ti)], the percentage growth was calculated at each of the drug concentration levels (Figure 4). Percentage growth inhibition was calculated as: $[(\mathrm{Ti}-\mathrm{Tz}) /(\mathrm{C}-\mathrm{Tz})] \times 100$ for concentrations having $\mathrm{Ti} \geq \mathrm{Tz}(\mathrm{Ti}-\mathrm{Tz})$ positive 
or zero, and $[(\mathrm{Ti}-\mathrm{Tz}) / \mathrm{Tz}] \times 100$ for concentrations having $\mathrm{Ti}<\mathrm{Tz}(\mathrm{Ti}-\mathrm{Tz})$ negative. The dose response parameters were calculated for each formulation. Growth inhibition of $50 \%\left(\mathrm{GI}_{50}\right)$ is the drug concentration resulting in a 50\% reduction in the net protein increase in control cells during the drug incubation and it was calculated from $[(\mathrm{Ti}-\mathrm{Tz}) /(\mathrm{C}-\mathrm{Tz})] \times 100=50$. The drug concentration resulting in total growth inhibition (TGI) was calculated from $\mathrm{Ti}=\mathrm{Tz}$. The $\mathrm{LC}_{50}$ (concentration of drug resulting in a $50 \%$ reduction in the measured protein at the end of the drug treatment as compared to initial) indicating a net loss of cells following treatment was calculated from $[(\mathrm{Ti}-\mathrm{Tz}) / \mathrm{Tz}] \times 100=-50$.

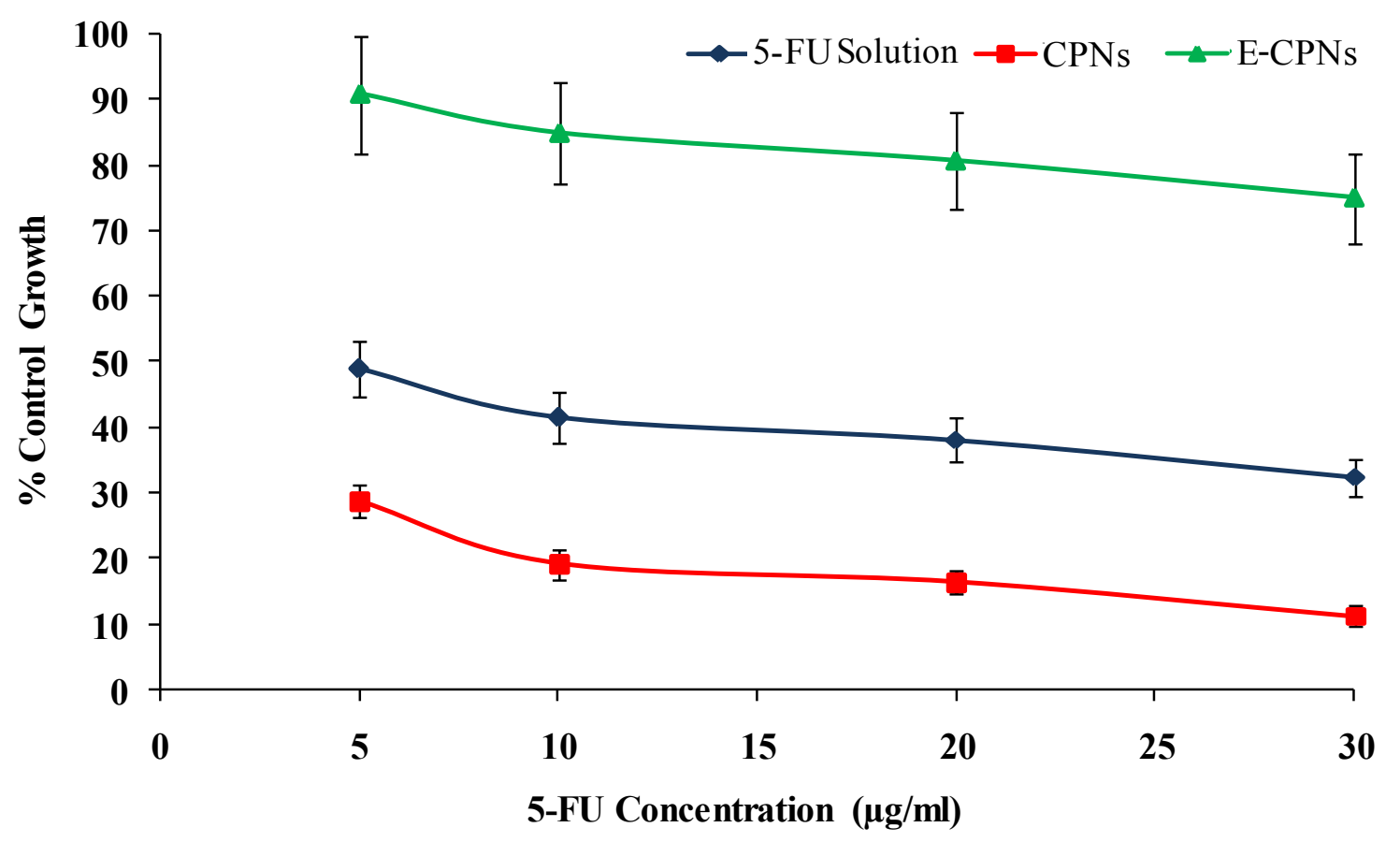

Figure 4. Cytotoxicity of 5-FU solution, CPNs and E-CPNs after $48 \mathrm{~h}$ incubation against HT-29 colorectal cancer cell lines (Mean $\pm \mathrm{SD}, n=3$ ).

\subsection{In vivo Studies}

\subsubsection{Experimental Protocol Approval}

Albino rats (Wistar strain) of either sex weighing about 120-150 grams were used for the present in vivo study. The experimental protocol was duly approved by the Institutional Animal Ethics Committee, Dr. H. S. Gour Vishwavidyalaya, Sagar (M.P.), India. The formulations were administered orally in equivalent doses to rats and a comparison for their organ distribution profiles was made. These animals were kept in well-spaced ventilated cages and maintained on healthy and fixed diets (Bengal grams soaked in water). They were fasted overnight before the experiment but allowed access to water ad libitum.

\subsubsection{Blood Profile and GI Organ Distribution Study}

A total of 36 animals were used for this study $(n=3)$. The animals were divided into four groups. Each group contained three animals. All animals were administered orally with the help of cannula, the formulation contained drug solution equivalent to $7.14 \mathrm{mg} / \mathrm{kg}$ body weight of the animal. Group 1 
received no drug and acted as control. Group 2 received 5-FU solution while Group 3 and Group 4 received CPNs and E-CPNs, respectively. After the administration of the drug, one animal from each group was sacrificed at each time interval i.e., $2 \mathrm{~h}, 4 \mathrm{~h}$, and $8 \mathrm{~h}$. Before sacrificing the animals, a blood sample $(0.1 \mathrm{~mL})$ was taken from the retro-orbital plexus using a uniformly tapered capillary at stated time points. Samples were collected in eppendorfs over $20 \mu \mathrm{L}$ heparin sodium $(22 \mathrm{mg} / \mathrm{mL})$ and centrifuged $(3000 \mathrm{~g}, 10 \mathrm{~min})$ at $4{ }^{\circ} \mathrm{C}$ to separate plasma [30]. Cold acetonitrile was added to these samples and centrifuged $(5000 \mathrm{~g}, 10 \mathrm{~min})$ at $4{ }^{\circ} \mathrm{C}$. The supernatants were collected and filtered through $0.22 \mu \mathrm{m}$ syringe filters and concentrations were determined by an HPLC method [31]. The mobile phase consisted of methanol: water $(10: 90, \mathrm{v} / \mathrm{v})$ adjusted to $\mathrm{pH} 3.2$ with perchloric acid at a flow rate of $1.0 \mathrm{~mL} / \mathrm{min}$. The HPLC column was a C18 $(4.6 \mathrm{~mm} \times 250 \mathrm{~mm}, 5 \mu \mathrm{m})$ and the results are shown in Figure 5. For GI organ distribution studies, GI tract (GIT) was removed and the mesenteric and fatty acid tissues were separated. The GIT was segmented into the stomach, small intestine, caecum and colon. The luminal contents were removed by applying gentle pressure with wet scissors to the tissues. Organs and luminal contents were weighed. The GIT parts were cut open longitudinally and rinsed with PBS (pH 7.4) to remove any remaining luminal contents. The entire tissue part of each organ was homogenized with PBS ( $\mathrm{pH}$ 7.4) using tissue homogenizer (MAC Micro Tissue Homogenizer, New Delhi, India) at $4{ }^{\circ} \mathrm{C}$. Either $1 \mathrm{~g}$ of each organ or the whole GIT part/tissue was used in the case if the GIT part was found to be less than $1 \mathrm{~g}$. To the tissue homogenates, an equal volume of acetonitrile was added and mixed with vortex (30 s). Then, the tissue homogenates were centrifuged (2000g, $10 \mathrm{~min}$ ). The fatty layer was discarded and supernatants were collected. Luminal contents were diluted with PBS (pH 7.4) followed by centrifugation, and supernatants were collected. Supernatants from all the above samples were filtered through a $0.45 \mu \mathrm{m}$ membrane filter, and analyzed for 5-FU using the HPLC method [31]. The amount of drug recovered in different segments of the GI tract at different time intervals was calculated and is presented in Figure 6.

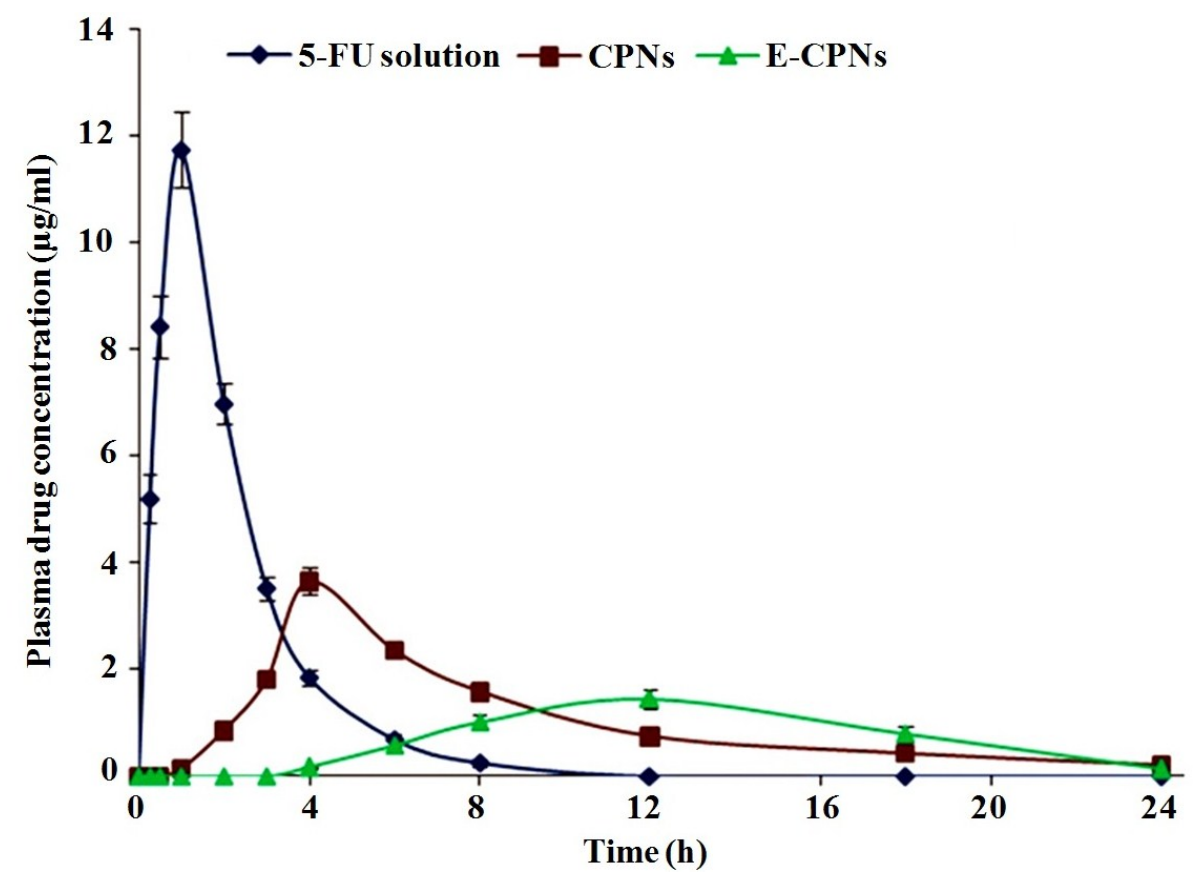

Figure 5. Mean plasma 5-FU concentration $v s$. time profile in albino rats after oral administration of various formulations (dose $\sim 7.14 \mathrm{mg} / \mathrm{kg}$ ) (Mean $\pm \mathrm{SD}, n=3$ ). 


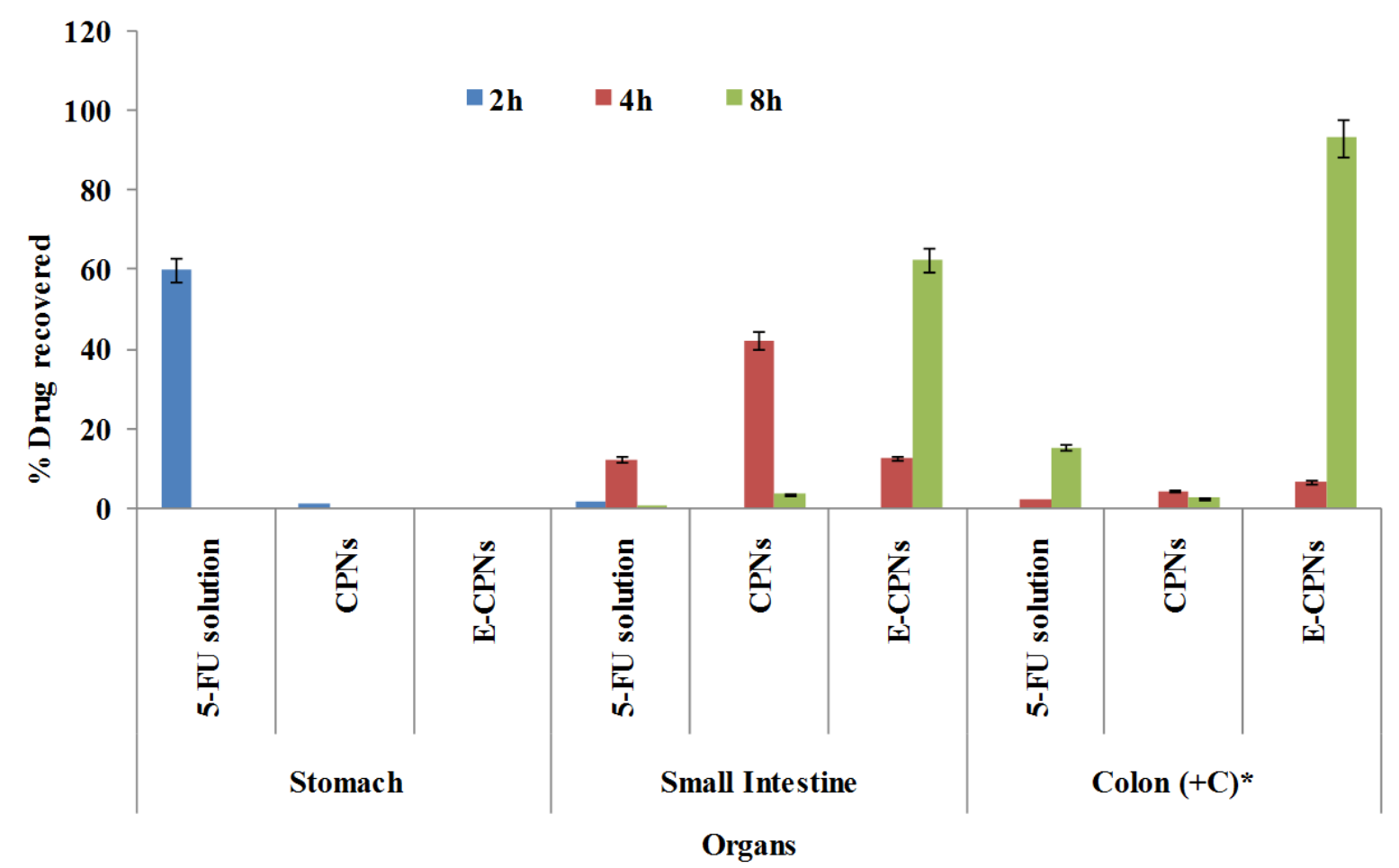

Figure 6. Distribution profiles of various formulations in different parts of GIT as a function of time (Mean $\pm \mathrm{SD}, n=3$ ).

\section{Statistical Analysis}

All results were expressed as mean \pm standard deviation (SD) and statistical analysis was performed with NCSS 2007 Version 07.1.14 (Kaysville, UT, USA). A difference with $p \leq 0.05$ (i.e., 5\%, level of significance) was considered to be statistically significant.

\section{Results and Discussion}

In the present work, a natural polymer i.e., citrus pectin was used to prepare the nanoparticles (CPNs and E-CPNs) bearing 5-FU. For rendering protection against the hostile gastric environment, Eudragit S100 was used to coat the CPNs meant for targeting colorectal cancer. As a polyanion natural polymer, pectin shows ability to crosslink in the presence of $\mathrm{Ca}^{2+}$ ions. CPNs were prepared using the method reported by Yu et al. (2009) [20] and relatively low concentration of $\mathrm{Ca}^{2+}$ was used to avoid complete gelation and formation of bulk gels. The alkaline system (pectin chains remained in stretched conformation) was dialyzed in water to bring the $\mathrm{pH}$ to neutral. Nanoparticles were spherical in shape with nanometric size and prominent integrity as shown in Figure 1. Average particle size of CPNs was $174.65 \pm 5.32 \mathrm{~nm}$ while E-CPNs were of $218.12 \pm 10.25 \mathrm{~nm}$ in size (Table 1). The zeta potential of the formulations can make a prediction about the stability of colloid dispersions and usually a high zeta potential $(>|30| \mathrm{mV})$ can provide an electric repulsion to avoid aggregation of particles. The zeta potential of CPNs was found to be negative i.e., $-18.4 \pm 0.4 \mathrm{mV}$ due to partial saturation of free galacturonic acid groups of pectin by $\mathrm{Ca}^{2+}$ ions whereas E-CPNs showed $-27.5 \pm 0.8 \mathrm{mV}$ zeta potential attributed to the presence of free acrylic acid groups on the surface. The percent entrapment efficiency of CPNs was determined after lysing the nanoparticles in PBS (pH 7.0) containing pectinase 
(120 FDU/mL). It was found to be $38.75 \% \pm 0.74 \%$ and $35.15 \% \pm 0.52 \%$ for CPNs and E-CPNs (Table 1).

In vitro drug release from the nanoparticles was assessed in simulated gastrointestinal fluid mediums of different $\mathrm{pH}$ according to the Souder and Ellenbogen extraction technique [23] using a modified USP dissolution test apparatus (Apparatus type 2). The in vitro release profile of E-CPNs (Figure 2) clearly exhibited negligible leaching of the drug at $\mathrm{pH} 4.5$ as compared to more than $20 \%$ drug release from CPNs.

In the case of CPNs, the release rate increased with increase in $\mathrm{pH}$. At $\mathrm{pH} 1.2$, the carboxyl groups of pectin are protonated, and the nanoparticles shrink and aggregate, leading to the slow release rate. As the $\mathrm{pH}$ increases, the number of negatively charged carboxylate groups increases and thus, the swelling ratio increases due to the ionization of carboxyl groups leading to repulsion in the pectin chains [32]. As a result, the release rate from nanoparticles increases with the increasing $\mathrm{pH}$ values of the release medium. The release data are in accordance with the fact that nanoparticles at SCF (pH 7.0) could have a high swelling ratio which leads to enhancement in permeability of the pectin matrix. The drug release was also favored by degradation of pectin due to pectinase (120 FDU/mL) which is found to be responsible for digestion of pectin in vivo. In the case of E-CPNs, there was no significant drug release up to $4 \mathrm{~h}$. Drug release began only after $4 \mathrm{~h}$ in simulated intestinal fluid (pH 6.8). This can be explained by the fact that the Eudragit S100 polymer contains carboxyl groups that ionize when the $\mathrm{pH}$ switches from acidic to alkaline. As the ionization takes place, the integrity of the coat is disturbed and the drug starts leaching from the nanoparticles [33]. In the case of CPNs, drug release was found to be $74.38 \% \pm 3.2 \%$ and $98.32 \% \pm 4.20 \%$ at the end of 8 and $24 \mathrm{~h}$, respectively while E-CPNs showed drug release of $57.64 \% \pm 2.1 \%$ and $79.24 \% \pm 3.15 \%$ at the end of 8 and $24 \mathrm{~h}$, respectively (Figure 2).

The in vitro drug release study of CPNs was also carried out in PBS ( $\mathrm{pH} 7.0$ ) with and without rat caecal content at $24 \mathrm{~h}$. The results showed $\sim 60 \%$ release of drug from CPNs opposed to $\sim 20 \%$ from E-CPNs in PBS ( $\mathrm{pH} 7.0$ ) without rat caecal content. While in the medium containing $2 \%$ rat caecal content, there were $\sim 92 \%$ and $\sim 60 \%$ drug release for CPNs and E-CPNs, respectively. The results showed improved drug release as compared to control (PBS pH 7.0) attributed to the various anaerobic bacteria present in caecum responsible for the digestion/degradation of the pectin facilitating release of drug from the nanoparticles (Figure 3) [34,35]. The controlled release profile of 5-FU from E-CPNs in our study corroborated well with those reported previously in addition to negligible release of 5-FU at lower $\mathrm{pH}(1-2)$ as compared to previously reported studies [36,37].

The SRB assay is based on the ability of the protein dye Sulforhodamine B to bind electrostatically in a $\mathrm{pH}$ dependent manner on protein basic amino acid residues of trichloroacetic acid-fixed cells. Under mild acidic conditions it binds, while under mild basic conditions it can be extracted from cells and solubilized for measurement [38]. Table 2 summarized the $\mathrm{LC}_{50}$, TGI and $\mathrm{GI}_{50}$ values of various formulations. Nanoparticles (CPNs and E-CPNs) showed significant cytotoxic effect and reduction in cell growth compared to free drug solution after $48 \mathrm{~h}$ incubation period in HT-29 cancer cell lines $(p<0.05)$. The amount of uncoated nanoparticles (CPNs) required to achieve $50 \%$ of growth inhibition $\left(\mathrm{GI}_{50}\right)$ was 1.5 fold lower than that of drug solution. This enhanced cytotoxic potential may be accounted for by the improved cellular internalization via galectin-3 receptors that over express in HT-29 colorectal cancer cell line [39]. Additionally, it could be due to anti-proliferative activity of citrus pectin by activation of caspase-3 pathway [40,41]. In the case of coated nanoparticles (E-CPNs), 
the cytotoxicity was significantly reduced as compared to CPNs which might be due to a number of reasons: (1) Firstly, Eudragit S100 having no self cytotoxicity potential on cell lines like HT-29, VK2, HeLa as reported by Yoo et al. (2011) [42]; (2) Secondly, the enteric coating unable to dissolve at the acidic $\mathrm{pH}$ of the medium used in the cell cytotoxicity study. However, little cytotoxicity of E-CPNs could be attributed to nominal leaching of drug over a period of $48 \mathrm{~h}$ of incubation. These results demonstrated that the incorporation of the drug in CPNs significantly enhanced the cytotoxic potential of the drug. Moreover, enteric coating successfully protected the nanoparticles in an acidic environment which was useful to establish in vitro-in vivo correlation. The growth curves exhibited control of growth in a concentration dependent manner in all formulations but CPNs showed superiority over the 5-FU control (Figure 4).

Table 2. Cytotoxicity potentials (SRB assay) of nanoparticles in HT-29 cancer cell lines.

\begin{tabular}{cccc}
\hline Formulation & $\mathbf{L C}_{\mathbf{5 0}}(\boldsymbol{\mu g} / \mathbf{m L})$ & TGI $(\boldsymbol{\mu g} / \mathbf{m L})$ & $\mathbf{G I}_{\mathbf{5 0}}(\boldsymbol{\mu g} \mathbf{g} \mathbf{m L})$ \\
\hline 5-FU solution & $56.7 \pm 4.2$ & $33.7 \pm 2.4$ & $9.3 \pm 0.7$ \\
CPNs & $36.4 \pm 3.2$ & $25.8 \pm 1.8$ & $6.5 \pm 0.4$ \\
E-CPNs & $94.2 \pm 3.8$ & $76.4 \pm 2.2$ & $18.5 \pm 0.5$ \\
\hline \multicolumn{4}{c}{ Values represent Mean $\pm \mathrm{SD}(n=3, p<0.05)}$.
\end{tabular}

In vivo studies are usually conducted to evaluate the site specificity of drug release and to obtain relevant pharmacokinetic information of the delivery system. Factors like $\mathrm{pH}$ of various biological fluids, enzyme system, and interaction of the dosage form with the biological environment are likely to influence the performance of the dosage form. In vitro cytotoxicity assay was carried out with an aim to establish the superiority of the developed nanoparticles, if any, over the 5-FU control. Since, the developed nanoparticles were intended for use against colorectal cancer, HT-29 human colon cancer cell line was chosen for the assay. The in vivo biological performance i.e., the GI distribution of any drug loaded pectin nanoparticles has not been previously reported. Although some studies relating to the biodistribution of pectin microspheres and beads in mice have been reported $[36,43,44]$. CPNs and E-CPNs were evaluated for in vivo performance in albino rats (Wistar strain) to speculate the evidence on their clinical applications. The percent drug recovered in different parts of the GIT is graphically presented in Figure 5.

The maximum recovery of $\mathrm{FU}$ (i.e., $59.80 \% \pm 4.83 \%$ ) was observed after $2 \mathrm{~h}$ in the stomach following oral administration of 5-FU solution and in subsequent hours; much less drug reached the small intestine, and negligible amount of drug was found in the colon. Less drug release from CPNs in the small intestine and colon might be due to the shrinkage of CPNs in the acidic environment (gastric milieu) leading to a more compact structure and thereby restricting the release of drug. While in the case of coated nanoparticles (E-CPNs), release was more hindered due to the additional enteric coating of Eudragit S100. Nominal recovery of 5-FU from CPNs in the stomach was accounted to erosion of the adhered drug to the surface CPNs and a phenomenal shrinking of CPNs in acidic milieu.

In the small intestine region (with fluids), the maximum amount of drug was recovered after $4 \mathrm{~h}$ of oral administration of different formulations. The amounts of drug recovered after $4 \mathrm{~h}$ were $12.29 \% \pm 0.98 \%, 42.10 \% \pm 2.95 \%, 4.24 \% \pm 0.35 \%$ for 5 -FU solution, CPNs and E-CPNs, respectively (Figure 5). This might be attributed to the different release profiles of the formulations. In the case of 
5-FU solution, the maximum amount of drug might have been absorbed through the stomach wall to systemic circulation thereby a lesser amount would reach the small intestine. However, in the case of CPNs, a relatively greater amount was recovered from the small intestine which might be due to the following reasons. Firstly, restricted release of drug in acidic medium as described earlier thereby resulting in a greater amount reaching the small intestine. Secondly, the presence of abundant microflora $\left(\sim 10^{2}-10^{7}\right.$ counts/g) along the whole length of the small intestine might have facilitated the drug release by enzymatic degradation of pectin. E-CPNs, being guarded by Eudragit S100 cover, showed the least drug recovery from the anterior part of the small intestine as compared to other formulations but it probably exhibited release in the ileo-caecal region i.e., the posterior part of the small intestine which provided a favorable $\mathrm{pH}$ to uncover the nanoparticles from the coating. In subsequent hours, a lesser amount of drug was found in the small intestine which might be due to the drug absorption and transit through the intestine [36].

After oral administration of various formulations, the biodistribution results for the colon that included the caecal contents, caecum tissue, colon contents, and colon tissue are demonstrated in Figure 5. The maximum drug recovery was observed in the case of E-CPNs after $8 \mathrm{~h}$ showing the peak level of 5-FU i.e., $92.90 \% \pm 4.65 \%$ as compared to $15.09 \% \pm 1.88 \%$ for 5 -FU solution and $62.47 \% \pm 3.65 \%$ for CPNs. The results confirmed that the least amount of drug was lost in the upper GIT from E-CPNs attributed to the protective coat. Moreover, enhanced increase in 5-FU concentration in the colon in the case of E-CPNs was noticed because of facilitated degradation by the microflora in the colonic region [45]. There was enhanced accumulation of 5-FU at the colon i.e., $200.48 \mu \mathrm{g} 5-\mathrm{FU} / \mathrm{g}$ rat colon which was better than in previously reported studies [46].

In order to evaluate drug absorption, plasma drug profile was established in terms of the plasma concentration vs. time profiles of 5-FU after oral administration of various formulations to the rats $(7.14 \mathrm{mg} / \mathrm{kg})$ and is depicted in Figure 6. After oral administration of the 5-FU solution, the drug was detected rapidly in plasma in the initial hours, attributed to the higher permeability coefficient of 5-FU in the upper GIT. Thereafter, the drug-plasma concentration decreased quickly to undetectable levels after $8 \mathrm{~h}$. These results were in accordance with the reports published by Zinutti et al. (1998) [47] and Li et al. (2008) [30]. In the case of CPNs, delayed and slow absorption was observed as 5-FU might not have been released in the GI tract from where the drug is absorbed relatively at a faster rate. However, it was released in the small intestine from where the drug showed slow absorption due to low permeability [45]. In the case of E-CPNs, the maximum 5-FU level was reached after $12 \mathrm{~h}$ of oral administration and then gradually decreased over the next $12 \mathrm{~h}$, which indicated the prolonged residence time of the released drug in the colon with slow leaching of the drug to systemic circulation due to low permeability and compromised surface area $[48,49]$.

The colon, acts like a homogeneous reservoir to elicit slow and constant drug input which is beneficial to the cancer therapy in the case of drugs with short plasma half-lives. The steady low plasma drug concentrations in the case of E-CPNs can provide not only a safety benefit by reducing the magnitude of peak plasma drug levels [50] but also result in sustained drug exposure of cancer cells [51]. 


\section{Conclusions}

The conventional chemotherapeutic approach has not been found to be very effective in colorectal cancer as the drug molecule does not reach the target site in effective concentrations. Pectin could be a promising carrier material in colon-specific drug delivery systems as data of studies conducted showed that Eudragit S100 coated CPNs (E-CPNs) were safe and effective in vitro and in vivo studies to deliver 5-FU for the effective treatment of colorectal cancer. Citrus pectin possessing multiple features also acts as a ligand for galectin-3 receptors that are over expressed on colorectal cancer cells and was found to enhance the targeting potential of E-CPNs to cancer cells.

\section{Supplementary Information}

Supplementary materials can be accessed at: http://www.mdpi.com/1996-1944/8/3/0832/s1.

\section{Acknowledgments}

We are thankful to Biochem (Mumbai) and Degussa (Germany) for providing gift sample of 5-FU and Eudragit S100, respectively. The authors are also thankful to All India Institute of Medical Sciences (AIIMS), New Delhi for carrying out TEM and SEM studies. Authors namely Ankit Jain and Pooja Hurkat are obliged to the Council of Scientific and Industrial Research (New Delhi, India) for rendering a Senior Research Fellowship (SRF) and a Research Associateship (RA) to Ashish Jain, respectively.

\section{Author Contributions}

All authors contributed extensively to the work presented in this paper.

\section{Conflicts of Interest}

The authors report no conflicts of interest. The authors alone are responsible for the content and writing of the manuscript.

\section{References}

1. Chaurasia, M.; Chourasia, M.K.; Jain, N.K.; Jain, A.; Soni, V.; Gupta, Y.; Jain, S.K. Cross-linked guar gum microspheres: A viable approach for improved delivery of anticancer drugs for the treatment of colorectal cancer. AAPS PharmSciTech 2006, 7, E143-E151.

2. Dangi, R.; Hurkat, P.; Jain, A.; Shilpi, S.; Jain, A.; Gulbake, A.; Jain, S.K. Targeting liver cancer via ASGP receptor using 5-FU-loaded surface-modified PLGA nanoparticles. J. Microencapsul. 2014, 31, 479-487.

3. Hu, Y.; Jiang, X.; Ding, Y.; Ge, H.; Yuan, Y.; Yang, C. Synthesis and characterization of chitosan-poly (acrylic acid) nanoparticles. Biomaterials 2002, 23, 3193-3201.

4. Dou, H.; Jiang, M.; Peng, H.; Chen, D.; Hong, Y. Ph-dependent self-assembly: Micellization and micelle-hollow-sphere transition of cellulose-based copolymers. Angew. Chem. Int. Ed. 2003, 42, 1516-1519. 
5. Tang, M.; Dou, H.; Sun, K. One-step synthesis of dextran-based stable nanoparticles assisted by self-assembly. Polymer 2006, 47, 728-734.

6. BeMiller, J.N. An introduction to pectins: Structure and properties. In Chemistry and Function of Pectins; ACS Symposium Series-American Chemical Society: Washington, DC, USA, 1986; Volume 310, pp. 2-12.

7. Sriamornsak, P.; Sungthongjeen, S.; Puttipipatkhachorn, S. Use of pectin as a carrier for intragastric floating drug delivery: Carbonate salt contained beads. Carbohydr. Polym. 2007, 67, 436-445.

8. Mennini, N.; Furlanetto, S.; Maestrelli, F.; Pinzauti, S.; Mura, P. Response surface methodology in the optimization of chitosan-Ca pectinate bead formulations. Eur. J. Pharm. Sci. 2008, 35, 318-325.

9. Kumar, M.; Mishra, R.K.; Banthia, A.K. Development of pectin based hydrogel membranes for biomedical applications. Int. J. Plast. Technol. 2010, 14, 213-223.

10. Cabrera, J.C.; Cambier, P.; Cutsem, P. Drug encapsulation in pectin hydrogel beads-A systematic study of simulated digestion media. Int. J. Pharm. Pharm. Sci. 2011, 3, 292-299.

11. Birch, N.P.; Schiffman, J.D. Characterization of self-assembled polyelectrolyte complex nanoparticles formed from chitosan and pectin. Langmuir 2014, 30, 3441-3447.

12. Jain, A.; Gulbake, A.; Shilpi, S.; Jain, A.; Hurkat, P.; Jain, S.K. A new horizon in modifcations of chitosan: Syntheses and applications. Crit. Rev. Ther. Drug Carrier Syst. 2013, 30, 91-181.

13. Liu, L.; Fishman, M.L.; Kost, J.; Hicks, K.B. Pectin-based systems for colon-specific drug delivery via oral route. Biomaterials 2003, 24, 3333-3343.

14. Grant, G.T.; Morris, E.R.; Rees, D.A.; Smith, P.J.; Thom, D. Biological interactions between polysaccharides and divalent cations: The egg-box model. FEBS Lett. 1973, 32, 195-198.

15. May, C.D. Industrial pectins: Sources, production and applications. Carbohydr. Polymers 1990, 12, 79-99.

16. Kohn, R. Ion binding on polyuronates-alginate and pectin. Pure Appl. Chem. 1975, 42, 371-397.

17. Niwa, K.; Takaya, T.; Morimoto, T.; Takada, K. Preparation and evaluation of a time-controlled release capsule made of ethylcellulose for colon delivery of drugs. J. Drug Target. 1995, 3, 83-89.

18. Chourasia, M.K.; Jain, S.K. Pharmaceutical approaches to colon targeted drug delivery systems. J. Pharm. Pharm. Sci. 2003, 6, 33-66.

19. Leclere, L.; van Cutsem, P.; Michiels, C. Anti-cancer activities of ph-or heat-modified pectin. Front. Pharmacol. 2013, 4, 128.

20. Yu, C.-Y.; Cao, H.; Zhang, X.-C.; Zhou, F.-Z.; Cheng, S.-X.; Zhang, X.-Z.; Zhuo, R.-X. Hybrid nanospheres and vesicles based on pectin as drug carriers. Langmuir 2009, 25, 11720-11726.

21. Maestrelli, F.; Cirri, M.; Corti, G.; Mennini, N.; Mura, P. Development of enteric-coated calcium pectinate microspheres intended for colonic drug delivery. Eur. J. Pharm. Biopharm. 2008, 69, 508-518.

22. Chaurasia, M.; Chourasia, M.; Jain, N.K.; Jain, A.; Soni, V.; Gupta, Y.; Jain, S. Methotrexate bearing calcium pectinate microspheres: A platform to achieve colon-specific drug release. Curr. Drug Deliv. 2008, 5, 215-219. 
23. Souder, J.; Ellenbogen, W. Control of d-amphetamine sulphate sustained release capsule. Drug Stand. 1985, 26, 77-79.

24. Jain, A.; Jain, S.K.; Ganesh, N.; Barve, J.; Beg, A.M. Design and development of ligand-appended polysaccharidic nanoparticles for the delivery of oxaliplatin in colorectal cancer. Nanomedicine 2010, 6, 179-190.

25. Van den Mooter, G.; Samyn, C.; Kinget, R. The relation between swelling properties and enzymatic degradation of azo polymers designed for colon-specific drug delivery. Pharm. Res. 1994, 11, 1737-1741.

26. Momin, M.; Pundarikakshudu, K. In vitro studies on guar gum based formulation for the colon targeted delivery of sennosides. J. Pharm. Pharm. Sci. 2004, 7, 325-331.

27. Vichai, V.; Kirtikara, K. Sulforhodamine B colorimetric assay for cytotoxicity screening. Nat. Protoc. 2006, 1, 1112-1116.

28. Kalhapure, R.S.; Akamanchi, K.G. Oleic acid based heterolipid synthesis, characterization and application in self-microemulsifying drug delivery system. Int. J. Pharm. 2012, 425, 9-18.

29. Skehan, P.; Storeng, R.; Scudiero, D.; Monks, A.; McMahon, J.; Vistica, D.; Warren, J.T.; Bokesch, H.; Kenney, S.; Boyd, M.R. New colorimetric cytotoxicity assay for anticancer-drug screening. J. Natl. Cancer Inst. 1990, 82, 1107-1112.

30. Li, X.; Xu, Y.; Chen, G.; Wei, P.; Ping, Q. Plga nanoparticles for the oral delivery of 5-fluorouracil using high pressure homogenization-emulsification as the preparation method and in vitro/in vivo studies. Drug Dev. Ind. Pharm. 2008, 34, 107-115.

31. Alsarra, I.A.; Yassin, A.E.; Abdel-Hamid, M.; Alanazi, F.K.; Aljuffali, I.A. Direct UPLC-MS-MS validated method for the quantification of 5-aminolevulinic acid: Application to in vitro assessment of colonic-targeted oral tablets. J. Chromatogr. Sci. 2011, 49, 428-433.

32. Bajpai, A.; Shukla, S.K.; Bhanu, S.; Kankane, S. Responsive polymers in controlled drug delivery. Prog. Polym. Sci. 2008, 33, 1088-1118.

33. Sharma, V.K.; Jain, A.; Soni, V. Nano-aggregates: Emerging delivery tools for tumor therapy. Crit. Rev. Ther. Drug Carrier Syst. 2013, 30, 535-563.

34. Licht, T.R.; Hansen, M.; Bergström, A.; Poulsen, M.; Krath, B.N.; Markowski, J.; Dragsted, L.O.; Wilcks, A. Effects of apples and specific apple components on the cecal environment of conventional rats: Role of apple pectin. BMC Microbiol. 2010, 10, 13.

35. Kotla, N.G.; Gulati, M.; Singh, S.K.; Shivapooja, A. Facts, fallacies and future of dissolution testing of polysaccharide based colon-specific drug delivery. J. Controll. Release 2014, 178, $55-62$.

36. Paharia, A.; Yadav, A.K.; Rai, G.; Jain, S.K.; Pancholi, S.S.; Agrawal, G.P. Eudragit-coated pectin microspheres of 5-fluorouracil for colon targeting. AAPS PharmSciTech 2007, 8, 12.

37. Dutta, R.K.; Sahu, S. Development of a novel probe sonication assisted enhanced loading of 5 -FU in SPION encapsulated pectin nanocarriers for magnetic targeted drug delivery system. Eur. J. Pharm. Biopharm. 2012, 82, 58-65.

38. Voigt, W. Sulforhodamine B assay and chemosensitivity. In Chemosensitivity; Springer: Berlin/Heidelberg, Germany, 2005; pp. 39-48.

39. Bergman, M.; Djaldetti, M.; Salman, H.; Bessler, H. Effect of citrus pectin on malignant cell proliferation. Biomed. Pharmacother. 2010, 64, 44-47. 
40. Olano-Martin, E.; Rimbach, G.H.; Gibson, G.R.; Rastall, R.A. Pectin and pectic-oligosaccharides induce apoptosis in in vitro human colonic adenocarcinoma cells. Anticancer Res. 2002, 23, 341-346.

41. Cheng, H.; Li, S.; Fan, Y.; Gao, X.; Hao, M.; Wang, J.; Zhang, X.; Tai, G.; Zhou, Y. Comparative studies of the antiproliferative effects of ginseng polysaccharides on HT-29 human colon cancer cells. Med. Oncol. 2011, 28, 175-181.

42. Yoo, J.-W.; Giri, N.; Lee, C.H. Ph-sensitive eudragit nanoparticles for mucosal drug delivery. Int. J. Pharm. 2011, 403, 262-267.

43. Jain, S.K.; Jain, A.; Gupta, Y.; Ahirwar, M. Design and development of hydrogel beads for targeted drug delivery to the colon. AAPS PharmSciTech 2007, 8, E56.

44. Vaidya, A.; Jain, A.; Khare, P.; Agrawal, R.K.; Jain, S.K. Metronidazole loaded pectin microspheres for colon targeting. J. Pharm. Sci. 2009, 98, 4229-4236.

45. He, W.; Du, Q.; Cao, D.-Y.; Xiang, B.; Fang, F. Pectin/ethylcellulose as film coatings for colon-specific drug delivery: Preparation and in vitro evaluation using 5-fluorouracil pellets. PDA J. Pharm. Sci. Technol./PDA 2007, 61, 121-130.

46. Bose, A.; Elyagoby, A.; Wong, T.W. Oral 5-fluorouracil colon-specific delivery through in vivo pellet coating for colon cancer and aberrant crypt foci treatment. Int. J. Pharm. 2014, 468, 178-186.

47. Zinutti, C.; Barberi-Heyob, M.; Hoffman, M.; Maincent, P. In-vivo evaluation of sustained release microspheres of 5-FU in rabbits. Int. J. Pharm. 1998, 166, 231-234.

48. Haupt, S.M.; Rubinstein, A. The colon as a possible target for orally administered peptide and protein drugs. Crit. Rev. Ther. Drug Carrier Syst. 2002, 19, 499-551.

49. Hoffart, V.; Lamprecht, A.; Maincent, P.; Lecompte, T.; Vigneron, C.; Ubrich, N. Oral bioavailability of a low molecular weight heparin using a polymeric delivery system. J. Controll. Release 2006, $113,38-42$.

50. Gupta, E.; Vyas, V.; Ahmed, F.; Sinko, P.; Cook, T.; Rubin, E. Pharmacokinetics of orally administered camptothecins. Ann. N. Y. Acad. Sci. 2000, 922, 195-204.

51. Lalloo, A.; Chao, P.; Hu, P.; Stein, S.; Sinko, P.J. Pharmacokinetic and pharmacodynamic evaluation of a novel in situ forming poly(ethylene glycol)-based hydrogel for the controlled delivery of the camptothecins. J. Controll. Release 2006, 112, 333-342.

(C) 2015 by the authors; licensee MDPI, Basel, Switzerland. This article is an open access article distributed under the terms and conditions of the Creative Commons Attribution license (http://creativecommons.org/licenses/by/4.0/). 